\title{
Características geológicas, isotópicas y estructurales del depósito vetiforme Los Mangos, Antioquia-Colombia
}

\author{
Edwin Naranjo-Sierra ${ }^{{ }^{*}}$; Mauricio Alvarán-Echeverri ${ }^{2}$
}

DOI: http://dx.doi.org/10.18273/revbol.v40n1-2018006 @ @

Forma de citar: Naranjo-Sierra, E., y Alvarán-Echeverri, M. (2018). Características geológicas, isotópicas y estructurales del depósito vetiforme Los Mangos, Antioquia-Colombia. Boletín de Geología, 40(1), 93-108. Doi: 10.18273/revbol.v40n1-2018006.

\section{RESUMEN}

La mina Los Mangos es un depósito vetiforme encajado en una zona de cizalla sinestral con deformación frágildúctil que generó milonitización de la roca encajonante, estructuras sigmoidales, boudinage y contactos veta-caja reactivados con presencia de salbanda (gouge). Se observaron al menos dos etapas de mineralización: la primera caracterizada por Qz + Py \pm Au y la segunda etapa por Qz + Py + Cpy + Gn + telururos de Au-Ag (silvanita y hessita) + telururos de $\mathrm{Pb}$ (altaita) + telururos de $\mathrm{Au}$ (calaverita) + telururos de Hg (coloradoita). Las alteraciones hidrotermales y la asociación paragenética observada permite sugerir que especies como $\mathrm{H}_{2} \mathrm{~S}$ o $\mathrm{HS}^{-}$fueron dominantes en los fluidos mineralizantes y en consecuencia, el complejo $\mathrm{Au}(\mathrm{HS})_{2}^{-}$fue probablemente el ligante y el medio de transporte del Au. La presencia de texturas de recristalización tipo Bulging permitieron inferir condiciones de temperatura bajas $\left(<350^{\circ} \mathrm{C}\right)$ y condiciones de deformación frágil-dúctil. Los valores isotópicos de $\delta^{18} \mathrm{O}$ en moscovitas hidrotermales varían entre $+11,5 \mathrm{y}+12,9 \% o$, los valores de $\delta \mathrm{D}$ varían entre $-58,5 \mathrm{y}$ $-63,4 \%$ y los valores de azufre $\delta^{34} \mathrm{~S}$ en pirita varían entre $+3,0$ y $+1,0 \%$. Los valores de $\delta 18 \mathrm{O}_{\text {agua }}$ varían entre $+10,6 \%$ y $12,0 \%$ y los valores de $\delta \mathrm{D}_{\text {agua }}$ varían entre $-43,4 \%$ y $-38,5 \%$. Estos valores isotópicos de $\delta^{18} \mathrm{O}$, $\delta \mathrm{D}$ y $\delta^{34} \mathrm{~S}$ muestran una afinidad magmática y/o metamórfica para el fluido mineralizante. Las características geológicas del depósito, incluyendo control estructural, mineralogía, roca encajante, alteración hidrotermal, estilo de mineralización y composición isotópica de $\delta^{18} \mathrm{O}, \delta \mathrm{D}$ y $\delta^{34} \mathrm{~S}$, indican que el depósito en la mina Los Mangos es compatible con el modelo de depósitos de oro orogénico (Orogenic Gold Deposits) del subtipo: Depósitos de Filones Auríferos Hospedados en Granitoides (Granitoid-Hosted Lode-gold Deposits).

Palabras clave: Mina Los Mangos; oro orogénico; vetiforme; zona de cizalla; telururos; isótopos estables.

\section{Geological, isotopic and structural characteristics of Los Mangos lode gold type deposit, Antioquia-Colombia}

\begin{abstract}
Los Mangos is a shear-zone hosted lode gold type deposit with brittle-ductile deformation, which produced wallrock mylonitization, sigmoidal structures, boudinage and reactivation of vein-wallrock contact. At least two mineralization events were identified: $\mathrm{Qz}+\mathrm{Py} \pm \mathrm{Au}$ for the first stage and $\mathrm{Qz}+\mathrm{Py}+\mathrm{Cpy}+\mathrm{Gn}+\mathrm{Au}-\mathrm{Ag}$ tellurides (silvanite and hessite) + Pb tellurides (altaite) + Au tellurides (calaverite) + Hg tellurides (coloradoite) for the second stage. Hydrothermal alteration and paragenetic association suggest that $\mathrm{H}_{2} \mathrm{~S}$ or $\mathrm{HS}^{-}$were the predominant sulfur species in ore fluids and, in consequence $\mathrm{Au}(\mathrm{HS})_{2}^{-}$was likely the gold-transporting complex. Recrystallized quartz textures suggest low temperature $\left(<350^{\circ}\right)$ and brittle-ductile conditions. The $\delta^{18} \mathrm{O}$ values of hydrothermal muscovite are $+11,5$ to $+12,9 \%$, the $\delta \mathrm{D}$ values are $-58,5$ to $-63,4 \% o$ and the $\delta^{34} \mathrm{~S}$ values of pyrite are $+3,0$ and $+1,0 \%$. The $\delta 18 \mathrm{O}_{\text {water }}$ values are $+10,6 \% o$ to $+12,0 \% o$ and the $\delta \mathrm{D}_{\text {water }}$ values are $-43,4 \% o$ and $-38,5 \%$. These isotopic values are compatible with a magmatic and/or metamorphic origin for the ore fluids. These geological characteristics including structural controls, nature host-rock, hydrothermal alterations, mineralization style and isotopic composition of $\delta^{18} \mathrm{O}, \delta \mathrm{D}$ and $\delta^{34} \mathrm{~S}$ suggest that Los Mangos lode type deposit is compatible with the Orogenic Gold Deposits of Granitoid-Hosted Lode-gold Deposits subtype.
\end{abstract}

Keywords: Los Mangos mine; orogenic gold; lode; shear zone; tellurides; stable isotopes.

\footnotetext{
${ }^{1}$ Operadora Minera S.A.S., Medellín, Colombia. (*)edwin.naranjo@operadoraminera.com.co

${ }^{2}$ Departamento de Ciencias Geológicas, Universidad de Caldas, Manizales, Colombia.mauricio_alvaran@ucaldas.edu.co
} 


\section{INTRODUCCIÓN}

La mina Los Mangos se encuentra localizada al noreste del departamento de Antioquia, en el distrito minero de El Bagre, en la región del bajo cauca antioqueño. Una zona ampliamente reconocida por sus explotaciones oro aluvial y mineralizaciones de tipo vetiforme (Londoño et al., 2009; Sillitoe, 2008; Shaw, 2000; Naranjo-Sierra et al., 2016). Hacia el sector sur de este distrito, en las inmediaciones del municipio de El Bagre, la geología está dominada por rocas plutónicas de edad carbonífera pertenecientes al stock de El Carmen (Leal-Mejía, 2011). Varias manifestaciones vetiformes están hospedadas en este cuerpo ígneo intrusivo, explotadas mayoritariamente por trabajos mineros subterráneos artesanales, carentes de análisis y estudios geológicos. Este artículo presenta una caracterización geológica de una de las vetas principales al interior del distrito minero El Bagre; enfocándose en los controles estructurales de la mineralización, etapas mineralizantes, secuencia paragenética y alteraciones hidrotermales principales. Adicionalmente, plantea un posible origen del fluido mineralizante, interpreta el medio de transporte y depositación del oro y propone un modelo genético para el depósito vetiforme de AuAg presente en mina Los Mangos.

\section{AMBIENTE GEOLÓGICO}

El depósito vetiforme de Au-Ag en la mina Los Mangos, es la expresión más norte del sistema El Carmen propuesto por Londoño et al. (2009). La veta se encuentra encajada en rocas plutónicas carboníferas del stock El Carmen (Leal-Mejía, 2011; Varona-Bravo et al., 2016). A nivel regional la principal estructura al occidente es la falla Otú con una disposición NS a NNW cerca al municipio de Zaragoza, con un movimiento interpretado como sinestral, que pone en contacto rocas plutónicas carboníferas del stock El Carmen con rocas metamórficas permo-triásicas agrupadas regionalmente como Complejo Cajamarca (Londoño et al., 2009). La falla Otú es la expresión más septentrional del sistema de fallas Otú-Pericos la cual limita el terreno Chibcha (Ch) del terreno Tahamí (Th) de acuerdo a Restrepo y Toussaint (1988). $\mathrm{Al}$ este se encuentra la falla El Bagre interpretada regionalmente como una falla de cabalgamiento, que pone en contacto al batolito de Segovia con rocas metamórficas precámbricas agrupadas en el Gneis de San Lucas. Hacia el sureste se encuentran depósitos volcano-sedimentarios de Segovia y hacia el norte se encuentran las rocas sedimentarias de la Formación
Caucasia, la Formación Tarazá y los depósitos recientes de los ríos Nechí y Tigüí, que enmascaran la traza de la falla Otú y cubren discordantemente las rocas graníticas del batolito de Segovia (Londoño et al., 2009) y el stock El Carmen (FIGURA 1).

\section{GEOLOGÍA DEL DEPÓSITO}

La mineralización vetiforme de Au-Ag se encuentra encajada en el segmento más básico del stock El Carmen (Leal-Mejía, 2011; Varona-Bravo et al., 2016). Localmente, este cuerpo plutónico corresponde a una diorita melanocrática, de textura holocristalina fanerítica, tamaño de grano medio y una composición mineral simple: plagioclasa cálcica, anfíboles y en menor proporción cuarzo (Varona-Bravo et al., 2016) (FIGURA 2).

Geoquímicamente, el stock ElCarmen presenta contenidos de $\mathrm{SiO}_{2}$ entre 50-78\% (\% en peso), afinidad calcoalcalina, carácter metaluminoso a ligeramente peraluminoso (A/CNK $<1,1$ o $\left.\mathrm{Mol} \quad \mathrm{Al}_{2} \mathrm{O}_{3} / \mathrm{CaO}+\mathrm{Na}_{2} \mathrm{O}+\mathrm{K}_{2} \mathrm{O}<1,1\right)$, enriquecimiento de LREE (Light Rare Earth Elements: Sc, $\mathrm{La}, \mathrm{Ce}, \mathrm{Pr}, \mathrm{Nd}, \mathrm{Pm}, \mathrm{Sm}, \mathrm{Eu}, \mathrm{Gd}$ ) respecto a HREE (Heavy Rare Earth Elements: Y, Tb, Dy, Ho, Er, Tm, Yb, $\mathrm{Lu}$ ) y afinidad a granitos formados en arcos volcánicos continentales; todas estas características de granitos orogénicos oxidados (Takagi y Tsukimura, 1997; LealMejía, 2011; Varona-Bravo et al., 2016) (FIGURA 3).

Al interior de la mina Los Mangos, cerca al contacto con la veta, este cuerpo ígneo exhibe una textura milonítica con indicadores cinemáticos sinestrales (Varona-Bravo et al., 2016). También es común observar, fragmentos de roca de caja con alteración sericítica encapsulados al interior de la veta.

La mina Los Mangos es un depósito vetiforme en una zona de cizalla sinestral-inversa encajado en intrusivo y dominado composicionalmente por cuarzo, moscovita y calcita como minerales de alteración, pirita, sulfuros de metales base ( $\mathrm{Pb}$ y $\mathrm{Cu}$ ), oro y telururos de $\mathrm{Au}, \mathrm{Au}-$ Ag, $\mathrm{Pb}$ y Hg. Actualmente, esta operación subterránea tiene 3 niveles de explotación con una extensión en el sentido del rumbo de $1,5 \mathrm{~km}$, en sentido del buzamiento $200 \mathrm{~m}$ y un espesor promedio de veta de 1,0 m. La veta se encuentra desplazada métricamente (50 m promedio) en rumbo y buzamiento y está controlada por zonas de falla con carácter sinestral-inverso subverticales tipo Riedel (Starling, 2015) denominadas de norte a sur: Cambiavías, 35 sur, 170 norte, 440 norte, San Pedro y Mangos. 


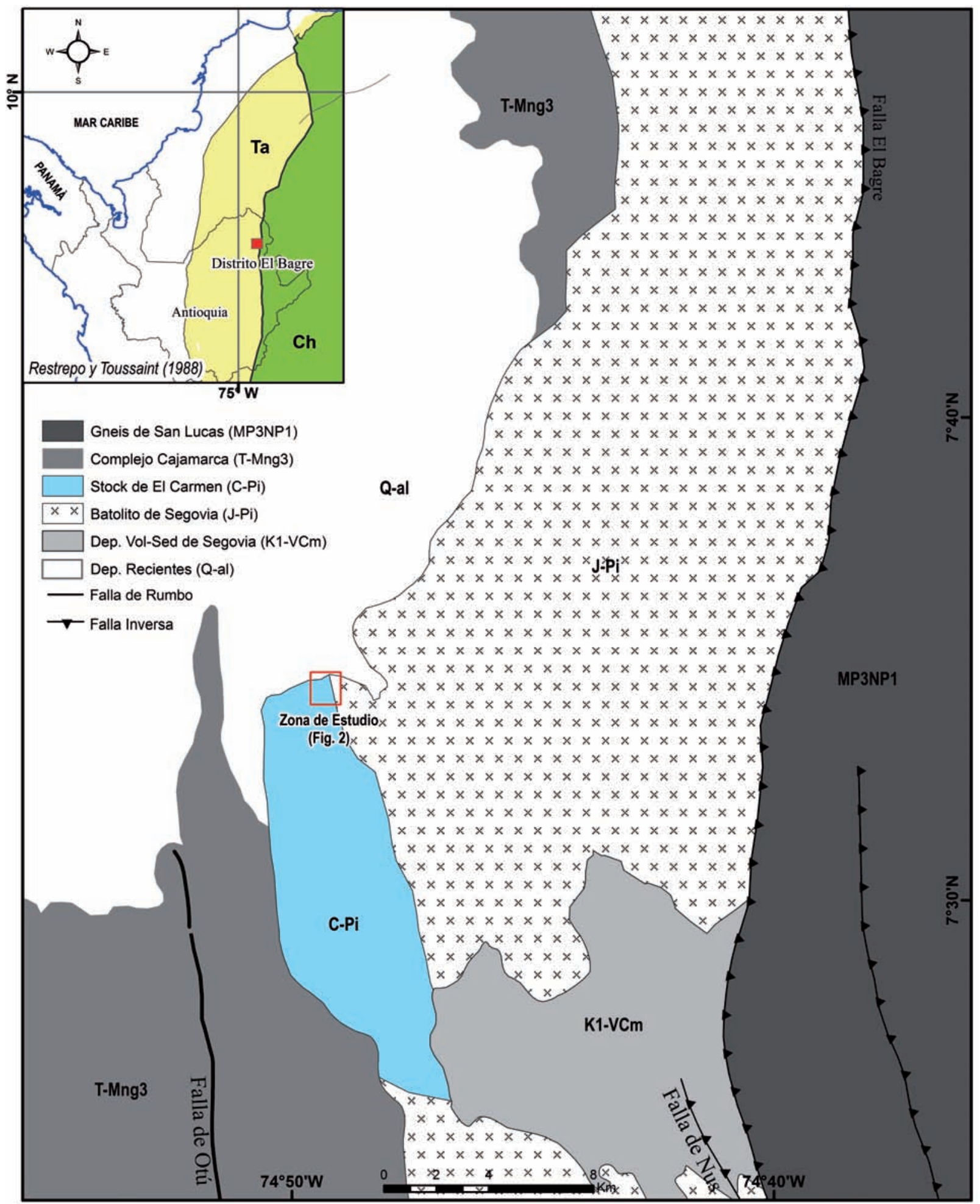

FIGURA 1. Mapa geológico regional de la zona de estudio. Terreno Chibcha (Ch), terreno Tahamí (Ta) y terreno Caribe (Ca) de acuerdo a Restrepo y Toussaint (1988). 


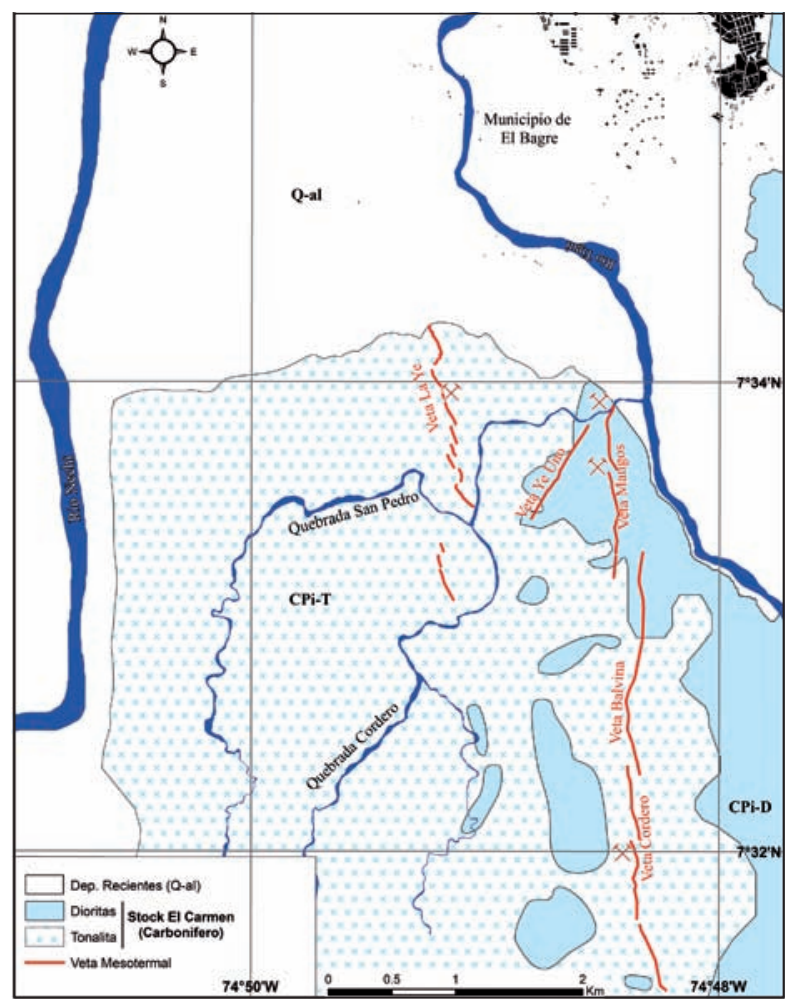

FIGURA 2. Mapa geológico local de la zona de estudio. Terreno Chibcha (Ch), terreno Tahamí (Ta) y terreno Caribe (Ca) de acuerdo a Restrepo y Toussaint (1988).
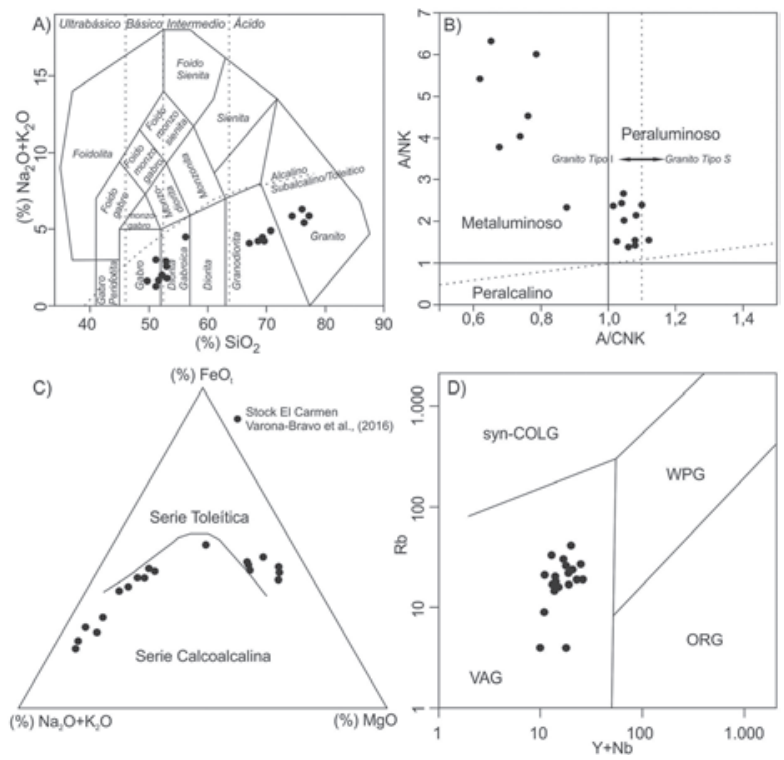

FIGURA 3. Diagramas geoquímicos del stock El Carmen. Tomado y modificado de Varona-Bravo et al. (2016). A. Clasificación química de las rocas de acuerdo a Middlemost (1994). B. Índice de Shand: $\mathrm{A} / \mathrm{CNK}$ (Mol $\mathrm{Al}_{2} \mathrm{O}_{3} /$ $\left.\mathrm{CaO}+\mathrm{Na}_{2} \mathrm{O}+\mathrm{K}_{2} \mathrm{O}\right)$ vs $\mathrm{A} / \mathrm{NK}$ ( $\left.\mathrm{Mol} \quad \mathrm{AL}_{2} \mathrm{O}_{3} / \mathrm{Na}_{2} \mathrm{O}+\mathrm{K}_{2} \mathrm{O}\right){ }^{2} \mathrm{de}$ acuerdo a Maniar y Piccoli (1989). C. Diagrama AFM propuesto por Irvine y Baragar (1971). D. Diagramas de discriminación tectónica de acuerdo a Pearce et al. (1984). Nota: syn-COLG (syn-collison granites), VAG (volcanic-arc granites), WPG (within-plate granites) y ORG (ocean-ridge granites).

\section{Geología estructural}

La veta se encuentra encajada en una zona de cizalla sinestral-inversa, con rumbo predominante NS y buzamiento al este (Starling, 2014; 2015) bajo un régimen de deformación frágil-dúctil, que generó milonitización de la roca caja, estructuras sigmoidales, boudinage y contactos veta-caja reactivados con presencia de salbanda (Londoño et al., 2009). Según Starling $(2014 ; 2015)$, la zona estuvo sometida a una compresión NNW/SSW, controlando los fluidos hidrotermales y la mineralización de metales base, oro y telururos, mediante fallamiento con tendencias promedio de $325^{\circ} / 85^{\circ} \mathrm{NE}$ tipo Riedel (ej. Cambiavías, 35 sur, 170 norte, 440 norte, San Pedro y Mangos). Adicionalmente, es posible observar eventos postmineralización con dos efectos opuestos: duplicación por cabalgamiento o segmentación por boudinage. Las vetas delgadas tienden a cizallarse a lo largo de los contactos y cuando las cizallas migran de piso a techo tienen un efecto de duplicación del espesor de la veta, interponiendo uno o más segmentos encima de la misma veta (cabalgamiento). Cuando el cizallamiento migra de techo a piso la veta tiende a separarse, generando adelgazamiento de la veta. Con vetas potentes, el cizallamiento tiende a estar activo a lo largo de ambos márgenes resultando en la formación de fracturas antitéticas estilo dominó que permiten al bloque de veta relativamente rígido rotar dentro de la zona de cizalla. El cizallamiento prolongado de la veta puede resultar en segmentos redondeados y separados (boudinage) formando una geometría en "rosario". Ambos escenarios son observados tanto en rumbo como en buzamiento (FIGURA 4).

\section{Mineralización y alteración hidrotermal}

Al menos dos etapas de mineralización fueron identificadas a través de análisis de microscopía óptica convencional complementados con microscopía electrónica de barrido (SEM-EDS). La primera etapa está caracterizada por volúmenes significativos de cuarzo acompañado por pirita y oro. La segunda y principal etapa de mineralización está conformada por sulfuros de metales base (calcopirita y en menor proporción galena), telururos de $\mathrm{Au}$ (calaverita), telururos de Au-Ag (silvanita y hessita), telururos de $\mathrm{Pb}$ (altaita), telururos de $\mathrm{Hg}$ (coloradoita) y oro. $\mathrm{La}$ presencia de telururos es la característica de esta etapa, y se constituyó en una guía de exploración debido a la relación directa entre Au-Te, como se observa en los análisis multielementales. Uniformemente distribuidos a lo largo de las exposiciones de la veta 
al interior de la mina, se observó moscovita y calcita como minerales de alteración hidrotermal dominantes. Microscópicamente la moscovita presenta formas alargadas subhedrales y tamaños que varían desde $3 \mu$ hasta $0,2 \mathrm{~mm}$. Los cristales de calcita generalmente se encuentran en venillas de $10-20 \mu$ de espesor o como acumulaciones pequeñas de cristales sin una distribución preferencial. La presencia de óxidos de hierro (goethita) está limitada a las exposiciones de veta más cercanas a la superficie, generalmente se presentan como pátinas que tiñen de color pardo rojizo la veta producto de alteración supergénica (FIGURA 5).
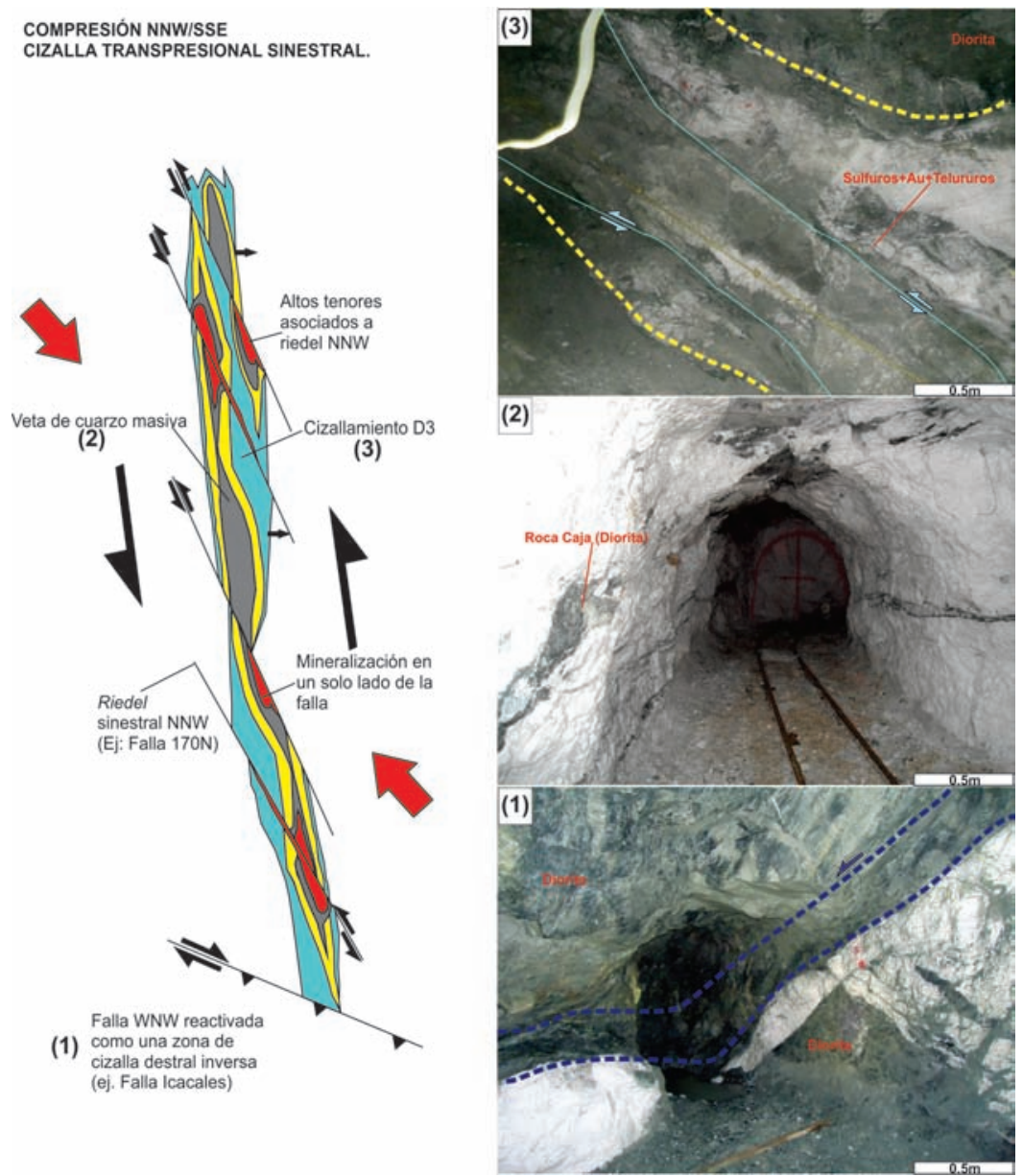

FIGURA 4. Esquema del modelo estructural en la mina Los Mangos, mostrando los controles estructurales y los estilos de la mineralización. Tomado y modificado de Starling (2015).

\section{Texturas de cuarzo y texturas de mena}

La veta en la mina Los Mangos fue emplazada y deformada dentro de una zona de cizalla bajo un régimen de deformación frágil-dúctil (Starling, 2014; 2015). El cuarzo es el mineral dominante en la veta, presenta texturas de recristalización dinámica tipo bulging, en donde la recristalización se da a lo largo de los contactos de los granos de cuarzo (Owona et al., 2013).

La pirita es el sulfuro dominante en la mineralización. Está caracterizada por presentar dos formas de presentación diferentes: la primera (py 1) está caracterizada por formas euhedrales, con tamaños de grano grueso, generalmente acompañada de $\mathrm{Au}$ y fuertemente fracturada. La segunda (py 2), presenta tamaños de entre $15-40 \mu$, con formas sub-redondeadas y está comúnmente asociada a galena, calcopirita y telururos. La presencia de metales base es común; se observó galena y calcopirita en formas anhedrales. Ambos generalmente acompañados de telururos. También es común observar los pits triangulares de la galena deformados. La presencia de telururos está asociada a la presencia de oro. Se identificaron telururos de $\mathrm{Au}, \mathrm{Au}-\mathrm{Ag}, \mathrm{Pb}$ y Hg, frecuentemente como relleno de fracturas en la pirita (py 1). 
Se identificaron claramente dos etapas de mineralización. La primera está caracterizada por la asociación pirita (py 1) y oro. Esta pirita del primer evento de depositación estuvo sometida a eventos de deformación, generando microfracturas en éste mineral, que posteriormente fueron selladas con minerales de la segunda etapa: calcopirita, galena, calaverita, silvanita, hessita, altaita, coloradoita, oro y pirita. Las texturas de mena entre estas especies minerales son generalmente de reemplazamiento y relleno de espacios abiertos. Las asociaciones y relaciones texturales entre los minerales definidos para ambos eventos, permitieron generar una secuencia paragenética (FIGURA 6).
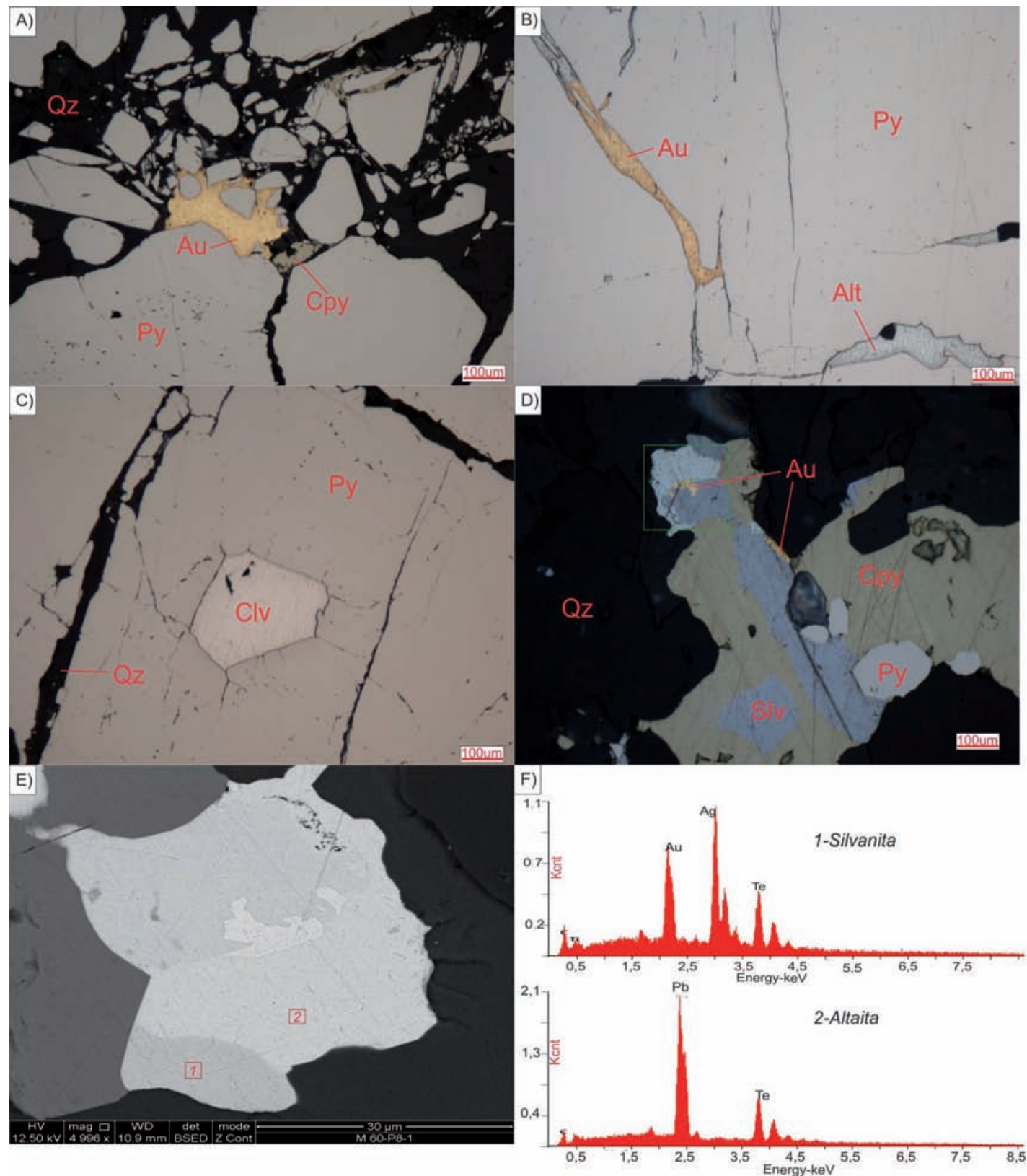

FIGURA 5. Características generales de la mineralización presente en la mina Los Mangos. A. Oro (Au) y calcopirita (Cpy) asociadas la etapa 2 (E2) llenando fracturas en pirita (Py) asociada a la etapa 1 (E1). 20X. NP. LR. B. Oro (Au) y altaita (Alt) asociadas a E2 llenando fracturas en pirita (Py) asociada a E1. 20X. NP. LR. C. Calaverita (Clv) asociada a la etapa 2 llenando fracturas de pirita (Py) asociada a la etapa 1. 20X. NP. LR. D. Silvanita (Slv), altaita (Alt), calcopirita (Cpy), oro (Au) y pirita (Py) asociados a la etapa 2. 20X. NP. LR. E. Imagen SEM del sector señalado en la FIGURA 6D, con silvanita (Slv), oro (Au) y altaita (Alt). BSED. 4.996x. 12,50kV. F. Espectros de análisis EDS (cuadros rojos en la FIGURA 6E) sobre silvanita (Slv) y altaita (Alt). NP (nicoles paralelos) y LR (luz reflejada). 


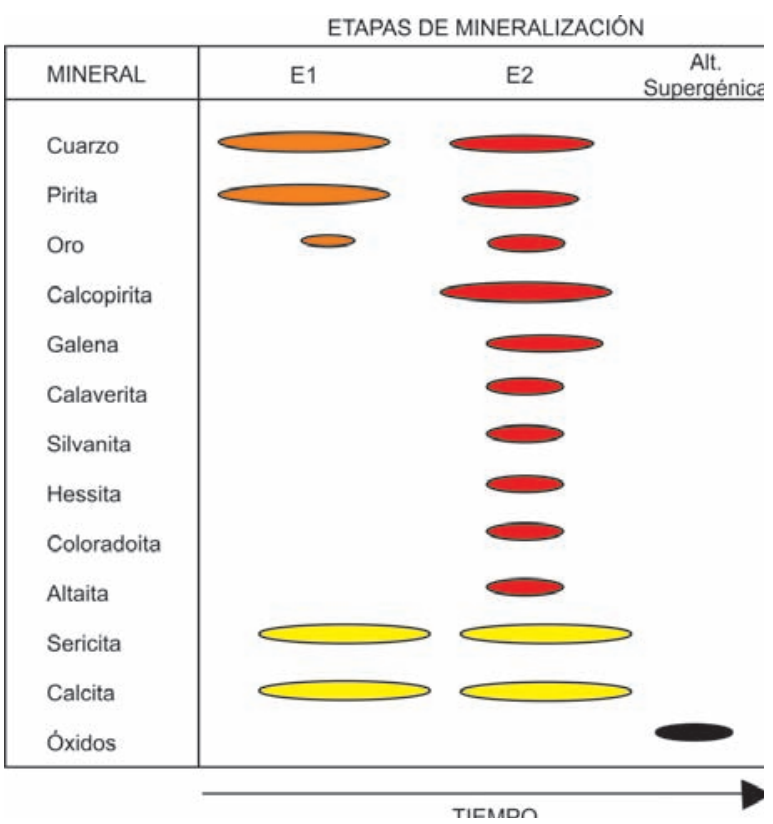

FIGURA 6. Secuencia paragenética de la mina Los Mangos con las etapas principales de mineralización. Espesor y continuidad de las líneas relativo a la abundancia durante cada evento de deformación.

\section{MATERIALES Y MÉTODOS}

Se llevó a cabo un muestreo sistemático y representativo del material de veta en los niveles $1 \mathrm{y}$ 2 de la mina Los Mangos. El análisis mineralógico, microscópico, paragenético y textural (i.e., cuarzo y minerales de mena) se llevó a cabo en el laboratorio de Yacimientos Minerales de la Universidad de Caldas en un microscopio petrográfico-metalográfico marca Nikon 200i Pol. El análisis de microscopía óptica de mena fue complementado con un análisis de microscopía electrónica de barrido (SEM-MEB) utilizando un equipo FEI QUANTA 250 con detectores BEI (Backscattered Electron Image) y SEI (Secundary Electron Image) para la generación de imágenes y detectores EDS (Energy Dispersive Spectrometer) y WDS (Wavelength Dispersive Spectroscopy) para análisis químico cualitativo y cuantitativo en muestras conductoras y no conductoras perteneciente al Instituto de Investigaciones en Estratigrafía (IIES) de la Universidad de Caldas. Adicionalmente se realizaron análisis multielementales (32 elementos) en el Laboratorio ACTLABS con tratamiento de agua regia y posterior análisis ICP-MS en un equipo Perkin Elmer Sciex ELAN 9000. Por último se complementó con análisis de isótopos estables de hidrógeno $(\delta \mathrm{D})$ y oxígeno $\left(\delta^{18} \mathrm{O}\right)$ en moscovita hidrotermal y azufre $\left(\delta^{34} \mathrm{~S}\right)$ en pirita realizados en el laboratorio ACTLABS, con el fin de determinar la naturaleza y posible fuente de los fluidos que dieron origen a la mineralización aurífera de la mina Los Mangos. Las muestras para el análisis de $\delta^{18} \mathrm{O}$ se hicieron reaccionar con $\mathrm{BrF}_{5}$ a $\sim 650^{\circ} \mathrm{C}$ en bombas de níquel siguiendo el procedimiento definido por Clayton y Mayeda (1963). Los análisis isotópicos de $\delta^{18} \mathrm{O}$ fueron realizados en un espectrómetro de masas Finnigan MAT. Las muestras para el análisis de $\delta \mathrm{D}$ se desgasificaron en vacío a $120^{\circ} \mathrm{C}$ durante 4 horas para eliminar el agua adsorbida en la superficie y se volvió a calentar inductivamente a $1.400^{\circ} \mathrm{C}$. El agua acumulada que representa la cantidad total de hidrógeno en las muestras se separó de los otros gases mediante técnicas diferenciales de congelación. El agua se hizo reaccionar con uranio a $900^{\circ} \mathrm{C}$ para producir $\mathrm{H}_{2}$ y se recogió en carbón a $-196^{\circ} \mathrm{C}$. El volumen de $\mathrm{H}_{2}$ se midió manométricamente. Los análisis isotópicos de $\delta \mathrm{D}$ fueron realizados en un espectrómetro de masas convencional. Los valores isotópicos de $\delta^{18} \mathrm{O}$ son reportados respecto a la notación delta estándar: $\delta^{18} O=$ $\left({ }^{18} \mathrm{O} /{ }^{16} \mathrm{O}\right)$ sample $\left.\left({ }^{18} \mathrm{O} /{ }^{16} \mathrm{O}\right)_{\text {vSMOW }}-1\right) 10^{3}$ per mil (\%o) de VSMOW (Vienna Standar Mean Ocean Water). La reproductibilidad externa es $\pm 0,19 \%$ o $(1 \sigma)$ con base en análisis repetidos de WCS (White Crystal Standard). Los valores de $\delta \mathrm{D}$ se reportaron en per mil (\%o) respecto al estándar VSMOW con reproductibilidad de los valores de $\pm 0,2 \%$ o, de acuerdo a la ecuación: $\delta \mathrm{D}=(\mathrm{D} / \mathrm{H})$ sample $\left./(\mathrm{D} / \mathrm{H})_{\text {VSMOW }}-1\right) 10^{3}$ per mil $(\%$ o de $V S M O W$. Las muestras para el análisis de $\delta^{34} S$ fueron quemadas a gas $\mathrm{SO}_{2}$. El $\mathrm{SO}_{2}$ es inyectado directamente a la fuente ionizada de un espectrómetro de masas VG602, siguiendo el procedimiento definido por Ueda y Krouse (1986). Los valores de $\delta^{34} S$ se reportan en per mil (\%o) respecto al estándar CDT (Canyon Diablo Troilite), con reproductibilidad de los valores mejores a $0,2 \%$, con base en la ecuación $\delta^{34} S=\left({ }^{34} S{ }^{2} S\right)$ sample/ $\left.\left({ }^{34} \mathrm{~S}^{2} \mathrm{~S}\right) C D T-1\right) 10^{3}$ per mil $(\% o)$.

\section{GEOQUÍMICA MULTIELEMENTAL}

Se procesaron 962 muestras con análisis multielementales de 32 elementos, realizados en diferentes exposiciones de veta al interior de la mina Los Mangos. La mineralización está caracterizada por las asociaciones $\mathrm{Au}-\mathrm{Te}$, Au-Ag-Te y $\mathrm{Au}-\mathrm{Te}-\mathrm{Pb}$ (TABLA 1). Las concentraciones de As, Se y Sb son bajas (promedio de 1,23 ppm, 0,53 ppm y 0,09 ppm respectivamente) descartando la presencia de arsenopirita, seleniuros y estibina en la mineralización. Las elevadas concentraciones de Te (>135 ppm), $\mathrm{Pb}(>115 \mathrm{ppm})$ y $\mathrm{Cu}(>181 \mathrm{ppm})$ corresponden a la presencia en la mineralización de telururos de $\mathrm{Au}$ (calaverita), telururos de Au-Ag (hessita y silvanita), telururos de $\mathrm{Pb}$ (altaita), galena y calcopirita 
respectivamente; introducidos durante la segunda y principal etapa mineralizante. Se observaron contenidos moderados de $\mathrm{Hg}(8,98 \mathrm{ppm})$ reflejando la presencia de coloradoita en la mineralización. Las concentraciones de W están cercanas al límite de detección $(<0,2 \mathrm{ppm})$. Las muestras analizadas presentan contenidos de $\mathrm{Au}$ y $\mathrm{Ag}$ variados, sin embargo la relación Au/Ag calculada con ensayes al fuego de más de 7.000 muestras de canal al interior de la mina Los Mangos varía entre 1:2 y 1:3. La buena correlación entre Au y Te es resultado de la presencia de telururos de $\mathrm{Au}$ y $\mathrm{Au}-\mathrm{Ag}$ en la mineralización (FIGURA 7).

TABLA 1. Matriz de correlación para las asociaciones de Au-Te, Au-Ag, Te-Pb, Te-Se y Te-Bi.

\begin{tabular}{|c|c|c|c|c|c|c|c|c|c|c|c|c|}
\hline & $A u$ & As & $T e$ & $B i$ & Se & $\mathrm{Hg}$ & $A g$ & $\mathrm{Cu}$ & $P b$ & $C d$ & $\mathrm{Zn}$ & $S b$ \\
\hline $\mathrm{Au}$ & 1,00 & & & & & & & & & & & \\
\hline As & 0,03 & 1,00 & & & & & & & & & & \\
\hline $\mathrm{Te}$ & 0,72 & 0,29 & 1,00 & & & & & & & & & \\
\hline $\mathrm{Bi}$ & 0,45 & 0,04 & 0,77 & 1,00 & & & & & & & & \\
\hline Se & 0,34 & 0,32 & 0,61 & 0,58 & 1,00 & & & & & & & \\
\hline $\mathrm{Hg}$ & 0,44 & 0,12 & 0,65 & 0,43 & 0,31 & 1,00 & & & & & & \\
\hline $\mathrm{Ag}$ & 0,80 & 0,09 & 0,78 & 0,48 & 0,45 & 0,46 & 1,00 & & & & & \\
\hline $\mathrm{Cu}$ & 0,08 & 0,08 & 0,32 & 0,38 & 0,58 & 0,25 & 0,15 & 1,00 & & & & \\
\hline $\mathrm{Pb}$ & 0,40 & 0,15 & 0,73 & 0,83 & 0,55 & 0,43 & 0,44 & 0,36 & 1,00 & & & \\
\hline Cd & 0,04 & 0,11 & 0,28 & 0,44 & 0,42 & 0,17 & 0,10 & 0,39 & 0,50 & 1,00 & & \\
\hline $\mathrm{Zn}$ & $-0,11$ & 0,14 & $-0,12$ & $-0,07$ & 0,17 & $-0,12$ & $-0,11$ & $-0,06$ & $-0,03$ & 0,12 & 1,00 & \\
\hline $\mathrm{Sb}$ & 0,34 & 0,25 & 0,52 & 0,54 & 0,48 & 0,31 & 0,40 & 0,33 & 0,53 & 0,33 & $-0,10$ & 1,00 \\
\hline
\end{tabular}

\section{RESULTADOS DE ISÓTOPOS ESTABLES}

Se realizaron análisis de isótopos estables de $\delta \mathrm{D}$, $\delta^{18} \mathrm{O}$ contenidos en filosilicatos de la veta y azufre $\delta^{34} \mathrm{~S}$ en pirita contenida en la veta TABLA 2. Todas las muestras fueron recolectadas al interior de la mina Los Mangos. La composición isotópica del hidrógeno $\delta \mathrm{D}$ y el oxígeno $\delta^{18} \mathrm{O}$, fue determinada en dos muestras usando procedimientos convencionales, de preparación y análisis; se seleccionaron y separaron moscovitas hidrotermales (moscovita). Los rangos de valores isotópicos obtenidos en este estudio en moscovita para $\delta^{18} \mathrm{O}$ están entre $+11,5$ y $+12,9 \%$ y para $\delta \mathrm{D}$ varían entre $-63,4$ y $-58,5 \%$ o respectivamente (FIGURA 8A). La mayoría de los granitos, rocas metamórficas y sedimentarias están enriquecidos en $\delta^{18} \mathrm{O}$ con relación al valor del manto, mientras que las aguas de mar y las aguas meteóricas tienen déficit de $\delta^{18} \mathrm{O}$ (Rollinson, 1993). Los valores anteriormente mencionados para oxígeno e hidrógeno proyectados en un diagrama de gráfico $\delta^{18} \mathrm{O} v s \delta \mathrm{D}$ grafican en el campo de aguas metamórficas según Taylor (1974) y Sheppard (1981), descartando de plano una mezcla con aguas meteóricas. La composición isotópica del azufre $\delta^{34} \mathrm{~S}$ (en pirita), fue determinada en dos muestras, pertenecientes a la segunda etapa de mineralización. Los valores varían entre $+1,0$ y $+3,0 \%$. Estos valores igualmente se podrían asociar a rocas graníticas y/o rocas metamórficas (FIGURA 8B).

\section{DISCUSIÓN}

Con los resultados obtenidos, se plantea el posible origen de los fluidos mineralizantes, se infiere la edad de la mineralización, se propone el medio de transporte y depositación del oro y se plantea un posible modelo genético para la mineralización presente en la mina Los Mangos, comparándolo con otros depósitos en la zona de estudio como el depósito de La Ye (NaranjoSierra et al., 2016), así como depósitos de referencia en Suramérica como son el depósito de Pataz en el Perú (Haeberlin, 2002) y Cachoeira en Brasil (Klein et al., 2005). 

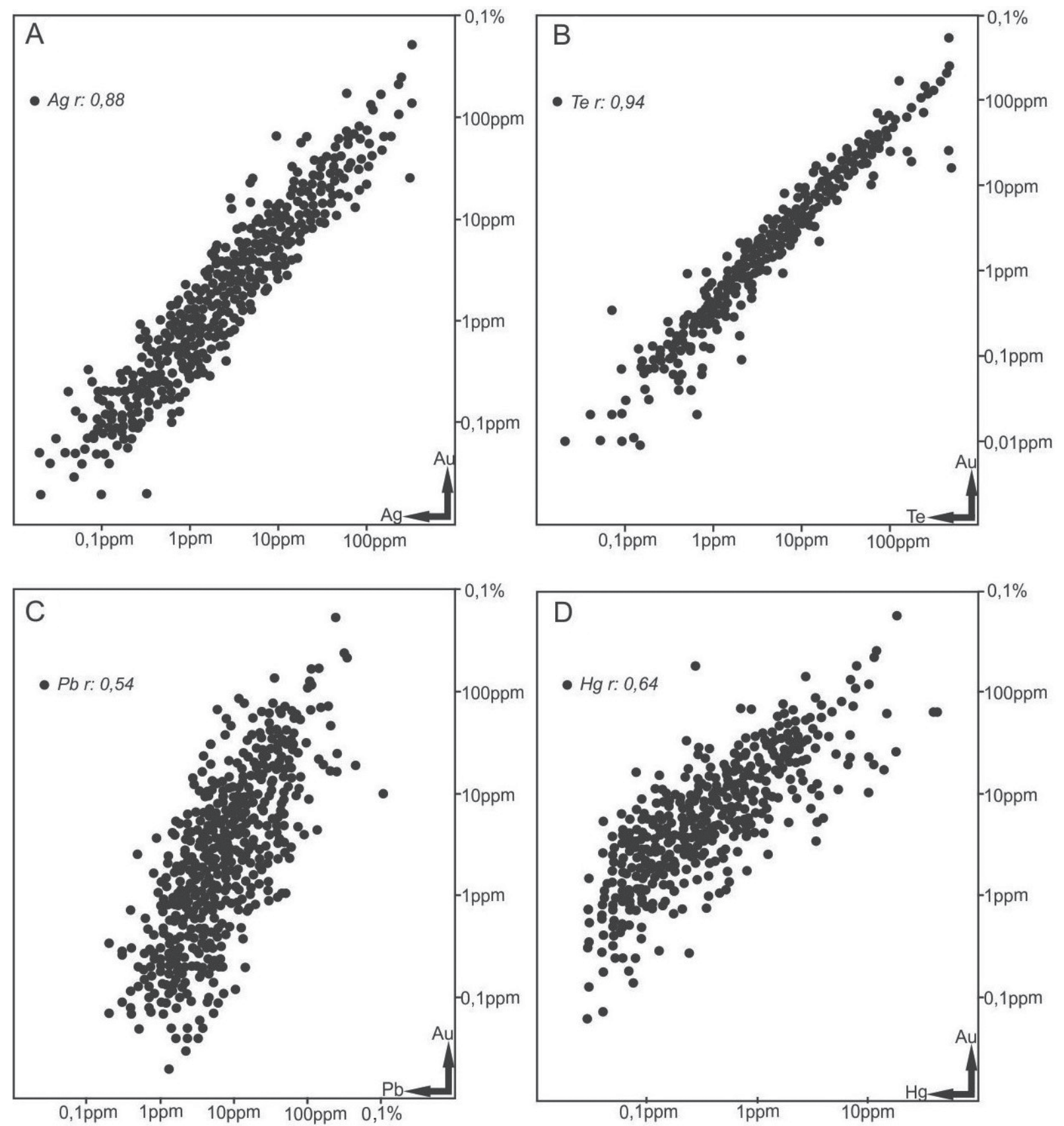

FIGURA 7. Gráficos bimodales. A. Au vs Ag. B. Au vs Te. C. Au vs Pb y D. Au vs Hg. Los coeficientes de correlación fueron calculados con el valor logarítmico de la concentración de cada elemento.

TABLA 2. Valores de isotopos de D, O y S en las muestras.

\begin{tabular}{lccccc}
\hline \multirow{2}{*}{ MUESTRA } & \multicolumn{2}{c}{$\delta \mathbf{D}(\% \mathbf{\%}, \mathbf{S M O W})$} & \multicolumn{2}{c}{$\boldsymbol{\delta}^{\mathbf{1 8}} \mathbf{O}(\mathbf{\%}, \mathbf{S M O W})$} & \multicolumn{2}{c}{$\delta^{\mathbf{3 2} \mathbf{S}^{\mathbf{c}}}$} \\
\cline { 2 - 6 } & Muscovita & $\mathbf{H}_{2} \mathbf{O}^{\mathbf{a}}$ & Muscovita & $\mathbf{H}_{\mathbf{2}} \mathbf{O}^{\mathbf{b}}$ & Pirita \\
\hline UCYE-25 & $-63,4$ & $-43,4$ & $+11,5$ & $+10,6$ & $+3,0$ \\
UCYE-28 & $-58,5$ & $-38,5$ & $+12,9$ & $+12,0$ & $+1,0$ \\
\hline
\end{tabular}

${ }^{a}$ El valor de $\delta^{18} \mathrm{O}_{\text {agua }}$ fue inferido de los valores $\mathrm{f}(f$-values) de acuerdo a Taylor (1997).

${ }^{\mathbf{b}}$ El valor de $\delta \mathrm{D}_{\text {agua }}$ fue estimado asumiendo un fraccionamiento entre agua y muscovita de $-20,0 \%$ a $330^{\circ} \mathrm{C}$ de acuerdo a las curvas-S (S-shapes curves) propuestas por Lambert y Epstein (1980).

${ }^{\mathrm{c}}$ Utilizando la siguiente aproximación: $\delta^{34} \mathrm{~S}_{\Sigma \mathrm{S}}=\delta^{34} \mathrm{~S}_{\mathrm{H} 2 \mathrm{~S}}=\delta^{34} \mathrm{~S}_{\mathrm{py}}$ para ${ }^{\circ} \mathrm{T}<500^{\circ} \mathrm{C}$ y pH $<6$ (Ohmoto y Goldhaber, 1997).

Boletín de Geología - Vol. 40 Num. 1 

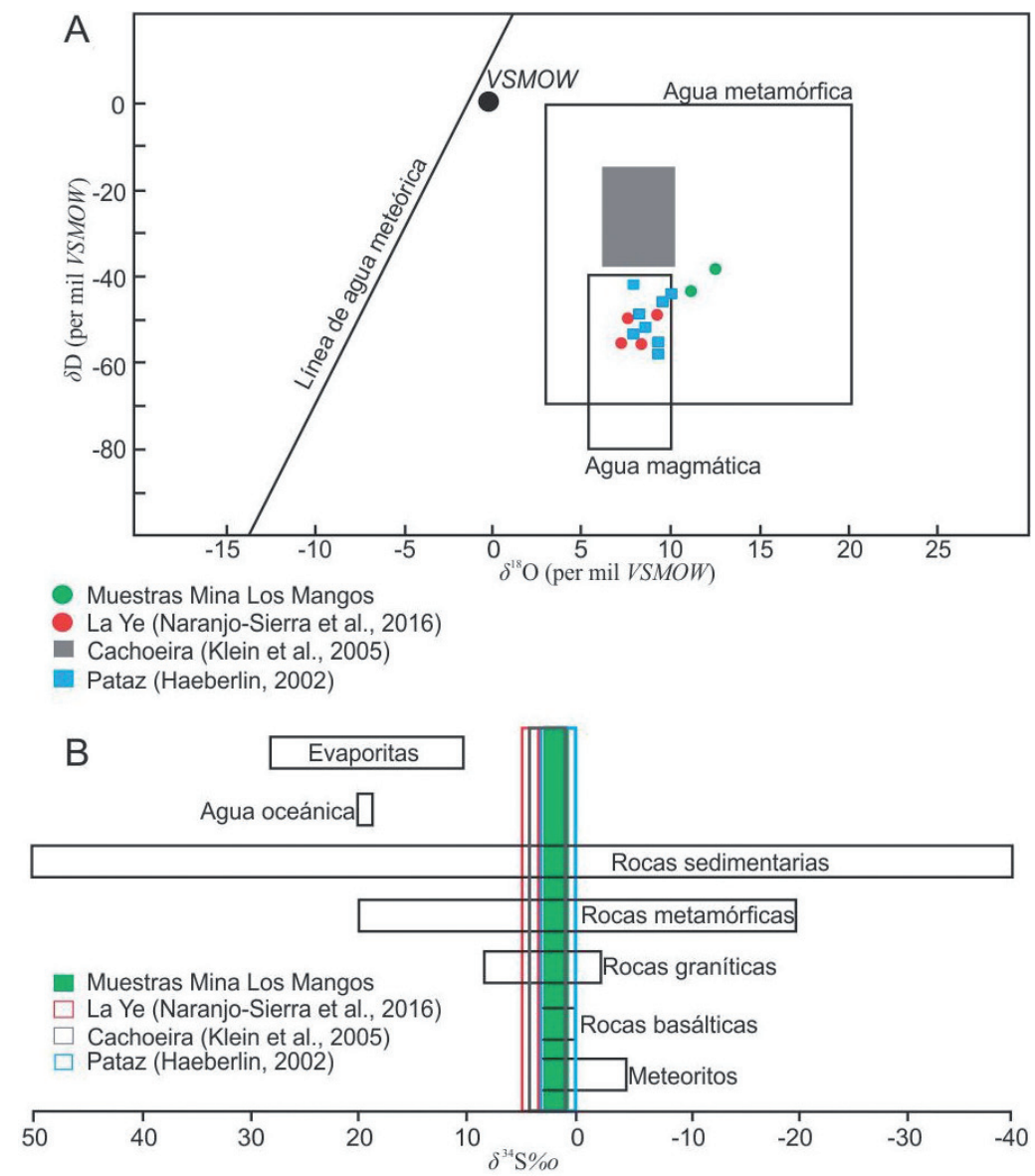

FIGURA 8. A. Valores de isótopos de oxígeno $\left(\delta^{18} \mathrm{O}\right)$ e hidrógeno $(\delta \mathrm{D})$ en muestras de moscovita en la mina Los Mangos. Se observan los campos de agua magmática (Taylor, 1974) y aguas metamórficas (Taylor, 1974; Sheppard, 1981). Los valores de $\delta^{18} \mathrm{O}$ y $\delta \mathrm{D}$ (en moscovita hidrotermal) calculados respecto a VSMOW están restringidos al campo de aguas metamórficas. B. Valores de isótopos de $\delta^{34} \mathrm{~S}$ en pirita, consistentes con los campos de rocas graníticas.

\section{Fuente de los fluidos mineralizantes}

Los resultados isotópicos para las muestras tomadas al interior de la mina Los Mangos, permiten evaluar preliminarmente la fuente del fluido mineralizante. Los valores $\delta^{34} \mathrm{~S}_{\mathrm{H} 2 \mathrm{~S}}$ del fluido hidrotermal pueden ser estimados directamente delos valores $\delta^{34}$ S delos sulfuros (pirita). La mineralización presente en la veta presenta especies de sulfuros reducidos y por consiguiente la composición isotópica del sulfuro total presente en el fluido $\left(\delta^{34} \mathrm{~S}_{\Sigma \mathrm{S}}\right)$ se podría considerar equivalente a los valores de $\delta^{34} S_{\mathrm{H} 2 \mathrm{~S}}$ (Haeberlin, 2002) Utilizando la siguiente aproximación: $\delta^{34} \mathrm{~S}_{\Sigma \mathrm{S}}=\delta^{34} \mathrm{~S}_{\mathrm{H} 2 \mathrm{~S}}=\delta^{34} \mathrm{~S}_{\mathrm{py}}$ para ${ }^{\circ} \mathrm{T}<500^{\circ} \mathrm{C}$ y $\mathrm{pH}<6$ (Ohmoto y Goldhaber, 1997); los valores de $\delta^{34} S_{\Sigma \mathrm{S}}$ están agrupados entre $+1,0 \mathrm{y}$ +3,0 per mil para pirita asociada a la segunda etapa (etapa aurífera). Los rangos y variaciones en los valores de $\delta^{34} S_{\Sigma \mathrm{S}}$ obtenidos pueden ser considerados como de afinidad magmática dominante relativamente homogénea, con azufre producido directamente por magmas o por re-movilización de sulfuro de rocas magmáticas (McCuaig y Kerrich, 1998; Brown et al., 2003; Klein et al., 2005). Aunque los estudios de isótopos de azufre en rocas ígneas relacionadas a depósitos son muy limitados, pueden dar una base en los procesos de fusión parcial o de asimilación de las rocas encajantes (Seal, 2006). La composición isotópica de azufre del manto ha sido tradicionalmente considerada entre 0 y $\pm 2 \%$ (Seal, 2006; Rye, 2005). Los valores de $\delta^{34} S$ en depósitos como La Ye, Cachoeira y Pataz presentan valores de azufre similares a los obtenidos en la mina Los Mangos (FIGURA 8B). Ridley y Diamond (2000) muestran que los sulfuros en la mayoría de los depósitos orogénicos tienen valores de $\delta^{34} \mathrm{~S}$ en un rango restringido desde -3 hasta $+9 \%$ o, el valor de $\delta^{34} \mathrm{~S}$ de sulfuros precipitados de un fluido puede variar significativamente de acuerdo a la fugacidad de oxigeno $\left(f \mathrm{O}_{2}\right)$ y el $\mathrm{pH}$; sin embargo, los valores calculados de $\delta^{34} \mathrm{~S}_{\text {fluido }}$ están entre $-1 \mathrm{y}+8 \%$, superponiendo los valores de reservorios comunes de azufre, incluyendo las rocas magmáticas manto derivadas y el promedio 
de las rocas de la corteza. Adicionalmente, la presencia de Te en la mineralización es un buen indicativo de un fluido con afinidad magmática (Cook et al., 2009).

Las mediciones de $\delta^{18} \mathrm{O}$ y $\delta \mathrm{D}$ en minerales de alteración hidrotermal, proveen una evaluación indirecta de la composición isotópica del fluido mineralizante. Para estimaciones adicionales, se asumió una temperatura de formación de $330^{\circ} \mathrm{C}$ de acuerdo a las texturas de cuarzo observadas y la alteración hidrotermal dominante. Debido a que ninguna curva publicada moscovita-agua es apropiada a bajas temperaturas (Haeberlin, 2002), el valor de $\delta^{18} \mathrm{O}_{\text {agua }}$ a $330^{\circ} \mathrm{C}$ fue inferido de los valores $\mathrm{f}$ (f-values) de acuerdo a Taylor (1997). Utilizando estos valores, se estimó un $\Delta^{18} \mathrm{O}_{\text {mu-agua }}$ de $0,9 \% o$. El valor de $\Delta \mathrm{D}_{\text {mu-agua }}$ fue estimado asumiendo un fraccionamiento entre agua y moscovita de $-20,0 \%$ a $330^{\circ} \mathrm{C}$ de acuerdo a las curvas-S propuestas por Lambert y Epstein (1980). Con base en lo anterior, los valores de $\delta^{18} \mathrm{O}_{\text {agua }}$ varían entre 10,6 y 12,0 per mil y los valores de $\delta \mathrm{D}_{\text {agua }}$ varían entre -43,4 y 38,5 per mil. Estos valores están en el campo de aguas metamórficas (FIGURA 8A), definido por Taylor (1974). El depósito La Ye, presenta valores isotópicos similares a los obtenidos para la mina Los Mangos, indicando una posible relación genética del fluido para ambos depósitos debido a su cercanía geográfica (distanciadas $3,5 \mathrm{~km}$ en línea recta). Aunque los valores de $\delta^{18} \mathrm{O}$ y $\delta \mathrm{D}$ obtenidos en este estudio, indican una posible afinidad metamórfica para el fluido mineralizante, los valores isotópicos de $\delta^{34} \mathrm{~S}$ y la presencia de Te en la mineralización indican contrariamente un posible origen magmático. Estos resultados permiten inferir que posiblemente ambas fuentes fueron responsables de la liberación de fluidos y metales en el origen de la veta en la mina Los Mangos. Sin embargo, cualquiera de estas dos posibles fuentes es consistente para los depósitos de oro orogénico, donde se tienen tres fuentes principales para los fluidos mineralizantes entre las cuales se incluye un origen magmático y/o metamórfico (Groves et al., 1998; Groves et al., 2003; Goldfarb et al., 2005; Moritz, 2000; Jia et al., 2003; Ridley y Diamond, 2000).

\section{Transporte y depositación del oro}

La información mineralógica e isotópica disponible son evidencias del $\mathrm{pH}$ y la condición redox del fluido (Klein et al., 2005). Estos parámetros ayudan en la comprensión del transporte y depositación del Au en el sistema hidrotermal de la mina Los Mangos. La ausencia de hematita y sulfatos en el ambiente de depositación, la co-existencia de pirita y clorita en la alteración hidrotermal y los valores de $\delta^{34} \mathrm{~S}$ en sulfuros, indican una condición relativamente reducida para la mineralización aurífera en la mina Los Mangos (Klein et al., 2005). Estas condiciones fisico-químicas estimadas son compatibles con la presencia de Te en la mineralización. La concentración y posterior depositación de telururos ocurrió posiblemente por la acción de procesos de separación de fases (ej: mecanismos de válvula de falla) comunes en depósitos orogénicos, en donde el campo de estabilidad del $\mathrm{Au}$ y telururos de $\mathrm{Au}-\mathrm{Ag}$ se alcanza en condiciones epizonales (Cook et al., 2009).

La presencia de moscovita y calcita como minerales de alteración dominantes, definen una naturaleza cerca de neutral a ligeramente alcalina para el fluido mineralizante (Mikucki, 1998). En estas condiciones físico-químicas estimadas, especies como $\mathrm{H}_{2} \mathrm{~S}$ o $\mathrm{HS}^{-}$ son dominantes en los fluidos y en consecuencia, el complejo $\mathrm{Au}(\mathrm{HS})_{2}^{-}$fue probablemente el medio ligante y de transporte (Klein et al., 2005; Benning y Seward, 1996; Ridley y Diamond, 2000). La presencia de calcita sugiere que el fluido mineralizante fue rico en $\mathrm{CO}_{2}$. $\mathrm{La}$ remoción del $\mathrm{CO}_{2}$ de la solución por disociación, de acuerdo a la reacción $\mathrm{CO}_{2}+\mathrm{H}_{2} \mathrm{O}=\mathrm{H}^{+}+\mathrm{HCO}_{3}^{-}$, aumenta el pH y la actividad del carbonato en la solución, causando la precipitación de minerales carbonatados (Rimstidt, 1997; Pirajno, 1992). La roca caja cerca al contacto con la veta presenta un halo de alteración hidrotermal que varía desde unos pocos centímetros hasta $1 \mathrm{~m}$ de espesor; este halo está dominado por la cloritización y epidotización de anfíboles (VaronaBravo et al., 2016) indicando unas condiciones de $\mathrm{pH}$ casi neutro a ligeramente ácido (Corbett y Leach, 1997; Pirajno, 1992); resaltando además que los fluidos estuvieron en un equilibro relativo con la roca caja, generando así un halo de alteración restringido, poco evidente o conspicuo como lo son en otros sistemas hidrotermales (Goldfarb et al., 2005).

\section{Edad de la mineralización de oro}

A pesar de la ausencia de dataciones en la veta de la mina Los Mangos, las relaciones de corte observadas en las diferentes exposiciones al interior de la mina y a lo largo del distrito minero El Bagre, permiten sugerir una edad para la mineralización/alteración. Tradicionalmente, al cuerpo encajante de las diversas manifestaciones vetiformes en el distrito minero El Bagre, se le ha asignado una edad jurásica, asociándola en mapas regionales al batolito de Segovia (Cediel y Cáceres, 2000; Londoño et al., 2009). Sin embargo, dataciones geológicas recientes U-Pb (SHRIMP) realizadas por Leal-Mejía (2011) demostraron que este cuerpo, hospedante de la mineralización, presenta una edad carbonífera (322-310 Ma) claramente 
diferente a las rocas graníticas jurásicas del batolito de Segovia (Spikings et al., 2015). Con base en estos nuevos datos, se podría asignar una edad postcarbonífera para la mineralización de la mina Los Mangos. Adicionalmente, Leal-Mejía (2011) realizó una datación $\mathrm{K}-\mathrm{Ar}$ en moscovita de alteración hidrotermal al interior de la mina La Ye, arrojando una edad pérmico-triásica (280 $\pm 6 \mathrm{Ma})$. Debido a su cercanía geográfica y a que ambas vetas comparten no solo la misma roca caja (stock El Carmen) sino posiblemente el mismo fluido hidrotermal, de acuerdo a los resultados de isótopos estables, se podría sugerir una edad permo-triásica para la mineralización de esta mina. Esta edad coincide claramente con las primeras etapas del evento tectono-magmático del PérmicoTriásico (ca. 280-230 Ma) definido por Vinasco et al. (2006), existiendo la posibilidad de que, tanto la veta y la alteración hidrotermal en la mina La Ye como en la mina Los Mangos y probablemente también las diferentes manifestaciones vetiformes en el distrito minero fuesen introducidas durante este evento.

\section{Un modelo genético para el depósito de Au-Ag en la mina Los Mangos}

La mina Los Mangos es un depósito de Au-Ag hospedado en una zona de cizalla, con mineralización en vetas de cuarzo. Las características geológicas, descritas en este documento, incluyendo geología estructural, roca encajonante, alteración hidrotermal, estilo de mineralización y composición isotópica de $\delta^{18} \mathrm{O}, \delta \mathrm{D}$ y $\delta^{34} \mathrm{~S}$, indican que la mina Los Mangos es compatible con el modelo de depósitos de tipo Oro Orogénico (Orogenic Gold Deposits), definido por Groves et al. (1998), del subtipo depósitos de Filones Auríferos Hospedados en Granitoides (GranitoidHosted Lode-gold Deposits) en el sentido de Haeberlin (2002), Qiu et al. (2002), Haeberlin et al. (2004) y Kreuzer (2006). En la TABLA 3 se presenta una comparación de las características geológicas de la mina Los Mangos contra las características definidas por Groves et al. (1998) para los depósitos orogénicos, Naranjo-Sierra et al. (2016) para el depósito La Ye, Haeberlin (2002) para el depósito de Pataz en Perú y Klein et al. (2005) para el depósito de Cachoeira en Brasil. Debido a que la veta en la mina Los Mangos se encuentra encajada en una roca ígnea plutónica se podría pensar en una posible relación genética entre la estructura vetiforme y el plutón encajonante como en los depósitos de tipo Relacionados a Intrusivos (Instrusion-Related) en el sentido de Sillitoe y Thompson (1998). Sin embargo, se debe ser cauteloso en deducir cualquier relación genética entre los depósitos vetiformes de Au asociados u hospedados en intrusivos
(Haeberlin, 2002; Hart, 2005). Las diferencias en el estilo de mineralización, características geoquímicas, ambiente tectónico y posible edad de la mineralización permitieron descartar por el momento este tipo de modelo genético para la mina Los Mangos.

\section{CONCLUSIONES}

La veta en la mina Los Mangos, está emplazada en una zona de cizalla sinestral-inversa, NS con buzamiento variante entre $30^{\circ}-40^{\circ} \mathrm{E}$; de $1,5 \mathrm{~km}$ de largo por 2,0 $\mathrm{m}$ de ancho, con un espesor de veta promedio de $1,0 \mathrm{~m}$, formada bajo un régimen frágil-dúctil. La mineralización está controlada por fallamiento tipo Riedel. Esta mineralización fue introducida por lo menos en dos etapas claramente identificadas. La primera caracterizada por volúmenes significativos de sílice, pirita y oro. Posteriormente, fue introducida una segunda etapa mineralizante caracterizada por la presencia de telururos de $\mathrm{Au}, \mathrm{Au}-\mathrm{Ag}, \mathrm{Pb}$ y $\mathrm{Hg}$, representados por calaverita, hessita, silvanita, altaita y coloradoita respectivamente.

Las texturas de recristalización del cuarzo observadas en las muestras de veta permiten inferir condiciones de temperatura bajas $\left(<350^{\circ} \mathrm{C}\right)$ y condiciones de deformación frágil-dúctil (Stipp et al., 2002; Owona et al., 2013). Estas condiciones de depositación y deformación están de acuerdo con las asociaciones mineralógicas y las alteraciones hidrotermales presentes, así como las características estructurales del depósito.

Los valores obtenidos de $\delta^{18} \mathrm{O}, \delta \mathrm{D}$ y $\delta^{34} \mathrm{~S}$ y la presencia de $\mathrm{Te}$ en la mineralización indican una afinidad magmática y/o metamórfica para el fluido mineralizante, con poca o nula participación de fluidos de origen meteórico.

Las asociaciones mineralógicas observadas en la veta de la mina Los Mangos, así como el tipo de alteración hidrotermal indican una condición relativamente reducida para la mineralización y una naturaleza neutra a ligeramente alcalina para el fluido mineralizante, probablemente rico en $\mathrm{CO}_{2}$ debido a la presencia de carbonatos al interior de la veta. En estas condiciones físico-químicas estimadas, especies como $\mathrm{H}_{2} \mathrm{~S}$ o HS son dominantes en los fluidos y en consecuencia, el complejo $\mathrm{Au}(\mathrm{HS})_{2}^{-}$fue probablemente el medio de transporte del oro.

Las características geológicas del depósito en la mina Los Mangos definidas en este trabajo, como: el emplazamiento de la veta en una zona de cizalla con deformación frágil-dúctil, la composición mineralógica 
de la veta, los minerales de alteración hidrotermal, el estrecho halo de alteración hidrotermal en la roca caja, las características estructurales del depósito, las texturas de cuarzo y los valores isotópicos de $\delta^{18} \mathrm{O}, \delta \mathrm{D}$ y $\delta^{34} \mathrm{~S}$; indican que la mina Los Mangos es compatible con el modelo de tipo Oro Orogénico (Orogenic Gold
Deposits), definidos por Groves et al. (1998), del subtipo: Depósitos de Filones Auríferos Hospedados en Granitoides (Granitoid-Hosted Lode-gold Deposits) en el sentido de Haeberlin (2002), Qiu et al. (2002), Haeberlin et al. (2004) y Kreuzer (2006).

TABLA 3. Comparación de características geológicas seleccionadas entre el modelo de Depósitos de Au Orogénico, mina La Ye y otros depósitos en suramérica.

\begin{tabular}{|c|c|c|c|c|c|}
\hline Características & Depósitos Orogénicos ${ }^{a}$ & $\begin{array}{c}\text { Mina La Ye } \\
\text { (Colombia) }\end{array}$ & $\begin{array}{c}\text { Depósitos de Pataz } \\
\text { (Perú) }\end{array}$ & $\begin{array}{c}\text { Depósito de } \\
\text { Cachoeira }^{\mathrm{d}} \text { (Brasil) }\end{array}$ & $\begin{array}{c}\text { Mina Los Mangos } \\
\text { (Colombia) }\end{array}$ \\
\hline Edad & $\begin{array}{c}\text { Arqueano Medio a } \\
\text { Terciario con picos en } \\
\text { Paleoproterozoico y } \\
\text { Fanerozoico }\end{array}$ & Pre-jurásico? & Paleozoico Superior & Paleoproterozoico & Pre-Jurásico? \\
\hline $\begin{array}{l}\text { Ambiente } \\
\text { Tectónico }\end{array}$ & $\begin{array}{l}\text { Márgenes continentales } \\
\text { deformados, terrenos } \\
\text { acrecionados }\end{array}$ & $\begin{array}{c}\text { Terrenos } \\
\text { acrecionados }\end{array}$ & $\begin{array}{l}\text { Cerca a estructuras de } \\
\text { primer orden NNW }\end{array}$ & $\begin{array}{c}\text { Back-Arc deformado/ } \\
\text { margen continental }\end{array}$ & $\begin{array}{c}\text { Terrenos } \\
\text { acrecionados }\end{array}$ \\
\hline $\begin{array}{l}\text { Roca } \\
\text { Hospedante }\end{array}$ & $\begin{array}{l}\text { Principalmente rocas } \\
\text { volcánicas, rocas } \\
\text { intrusivas o secuencias } \\
\text { sedimentarias }\end{array}$ & $\begin{array}{c}\text { Granitoide } \\
\text { carbonífero-Stock } \\
\text { El Carmen? }\end{array}$ & $\begin{array}{l}\text { Granitoides } \\
\text { carboníferos }\end{array}$ & $\begin{array}{l}\text { Rocas intrusivas y } \\
\text { volcánicas máficas, } \\
\text { rocas volcánicas } \\
\text { félsicas, esquistos } \\
\text { grafitosos, filitas }\end{array}$ & $\begin{array}{c}\text { Diorita carbonífera- } \\
\text { Stock El Carmen? }\end{array}$ \\
\hline $\begin{array}{l}\text { Tiempo de } \\
\text { Mineralización }\end{array}$ & $\begin{array}{c}\text { Tectono-tardío, post pico } \\
\text { metamórfico }\end{array}$ & $\begin{array}{l}\text { Syn a tectono } \\
\text { tardío? }\end{array}$ & Post pico metamórfico & $\begin{array}{l}\text { Syn a tectono-tardío, } \\
\text { post pico metamórfico }\end{array}$ & Syn a tectono tardío \\
\hline $\begin{array}{l}\text { Estilo de } \\
\text { Mineralización }\end{array}$ & $\begin{array}{l}\text { Vetas, arreglos } \\
\text { vetiformes, } \\
\text { reemplazamiento de } \\
\text { rocas ricas en Fe }\end{array}$ & $\begin{array}{l}\text { Vetas en zonas de } \\
\text { cizalla. Régimen } \\
\text { frágil-dúctil }\end{array}$ & $\begin{array}{c}\text { Vetas de cuarzo en } \\
\text { zonas de deformación } \\
\text { frágil-dúctil }\end{array}$ & $\begin{array}{c}\text { Arreglos vetiformes y } \\
\text { diseminado cerca a la } \\
\text { roca hidrotermalmente } \\
\text { alterada }\end{array}$ & $\begin{array}{l}\text { Vetas en zonas de } \\
\text { cizalla. Régimen } \\
\text { frágil-dúctil }\end{array}$ \\
\hline Tenor de Au & $>5 g / T$ & $3-8 g / T$ & $5-30 \mathrm{~g} / \mathrm{T}$ & - & $1-5 g / T$ \\
\hline $\begin{array}{l}\text { Alteración } \\
\text { Hidrotermal }\end{array}$ & $\begin{array}{c}\text { Moscovita, Carbonatos y } \\
\text { Sulfuros de Fe }\end{array}$ & $\begin{array}{l}\text { Moscovita, clorita } \\
\text { y carbonatos }\end{array}$ & $\begin{array}{l}\text { sericitización, } \\
\text { cloritización, } \\
\text { piritización y } \\
\text { silicificación }\end{array}$ & $\begin{array}{c}\text { Dolomita, pirita, } \\
\text { arsenopirita+/- } \\
\text { moscovita y clorita }\end{array}$ & Moscovita y Calcita \\
\hline 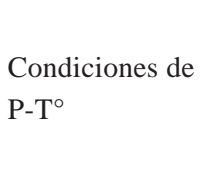 & $\begin{array}{c}\text { Principalmente } 350^{\circ}+/- \\
50^{\circ} \mathrm{C}, 1,5+/-0,5 \mathrm{kbar}\end{array}$ & $\begin{array}{c}\mathrm{T}^{\circ} \text { interpretada: } \\
\text { 300-350 C. P } \\
\text { desconocida }\end{array}$ & $\begin{array}{c}+/-300^{\circ} \mathrm{C} \text { y } 1-5 \mathrm{kbar} \\
\text { dependiendo } \\
\text { de la etapa de } \\
\text { mineralización }\end{array}$ & $\begin{array}{l}\text { 300-386 C. P } \\
\text { desconocida }\end{array}$ & $\begin{array}{c}\mathrm{T}^{\circ} \text { interpretada: } \\
\text { 300-350 } \mathrm{C} . \mathrm{P} \\
\text { desconocida }\end{array}$ \\
\hline $\begin{array}{l}\text { Composición del } \\
\text { Fluido }\end{array}$ & $\begin{array}{l}\text { Baja salinidad, } \mathrm{H}_{2} \mathrm{O}- \\
\mathrm{CO}_{2}+/-\mathrm{CH}_{4}+/-\mathrm{N}_{2}\end{array}$ & $\begin{array}{l}\text { Inferido: baja } \\
\text { salinidad } \\
\text { posiblemente } \\
\mathrm{H}_{2} \mathrm{O}-\mathrm{CO}_{2}\end{array}$ & $\begin{array}{c}\text { Baja salinidad. } \mathrm{H}_{2} \mathrm{O}- \\
\mathrm{NaCl} \text { y } \\
\mathrm{H}_{2} \mathrm{O}-\mathrm{NaCl}-\mathrm{CO}_{2}\end{array}$ & $\begin{array}{c}\text { Posiblemente } \\
\mathrm{H}_{2} \mathrm{O}-\mathrm{CO}_{2}\end{array}$ & $\begin{array}{l}\text { Inferido: baja } \\
\text { salinidad } \\
\text { posiblemente } \\
\mathrm{H}_{2} \mathrm{O}-\mathrm{CO}_{2}\end{array}$ \\
\hline Fuente de $\mathrm{O}$ y D & $\begin{array}{c}\text { Metamórfico y/o } \\
\text { Magmático }\end{array}$ & $\begin{array}{l}\text { Metamórfico y/o } \\
\text { Magmático }\end{array}$ & Metamórfico & Metamórfico & Metamórfico \\
\hline Fuente de S & Magmático & Magmático & $\begin{array}{c}\text { Magmático o de- } \\
\text { sulfidización de } \\
\text { minerales magmáticos } \\
\text { primarios }\end{array}$ & Magmático & Magmático \\
\hline $\begin{array}{l}\text { Tipo de } \\
\text { Depósito }\end{array}$ & & Orogénico & Orogénico & Orogénico & Orogénico \\
\hline
\end{tabular}

${ }^{\mathrm{a}}$ Groves et al. (1998 y 2003). ${ }^{\mathrm{b}}$ Naranjo-Sierra et al. (2016). ${ }^{\mathrm{c}}$ Haeberlin (2002). ${ }^{\mathrm{d}}$ Klein et al. (2005). ${ }^{\mathrm{e}}$ Este estudio. g/T: gramos/tonelada; kbar: kilobar; P: presión; D: deuterio; O: oxígeno 


\section{AGRADECIMIENTOS}

Queremos agradecer a Operadora Minera S.A.S por el acceso a la información y darnos la oportunidad de presentar este trabajo. También al grupo de exploración Greenfield y Brownfield quienes aportaron valiosos comentarios en el desarrollo de este estudio. Así mismo, agradecemos a Juan Carlos Molano y Alfonso Rodríguez Madrid por sus valiosos comentarios y recomendaciones en la revisión de este trabajo.

\section{REFERENCIAS}

Benning, L.G., and Seward, T.M. (1996). Hydrosulphide complexing of $\mathrm{Au}$ (I) in hydrothermal solutions from $150-400^{\circ} \mathrm{C}$ and 500-1500 bar. Geochimica et Cosmochimica Acta, 60(11), 1849-1871. doi: 10.1016/0016-7037(96)00061-0.

Brown, S.M., Johnson, C.A., Watling, R.J., and Premo, W.R. (2003). Constraints on the composition of ore fluids and implications for mineralising events at the Cleo gold deposit, Eastern Goldfields Province, Western Australia. Australian Journal of Earth Sciences, 50(1), 1938. doi: 10.1046/j.1440-0952.2003.00971.x.

Cediel, F., and Cáceres, C. (2000). Geological Map of Colombia. Third Ed. Geotec Ltd., Bogotá.

Clayton, R.N., and Mayeda, T. (1963). The use of bromine pentafluoride in the extraction of oxygen from oxides and silicates for isotopic analysis. Geochimica et Cosmochimica Acta, 27(1), 43-52. doi: 10.1016/0016-7037(63)90071-1.

Cook, N.J., Ciobanu, C.L., Spry, P.G., and Voudouris, P. (2009). Understanding gold-(silver)-telluride(selenide) mineral deposits. Episodes, 32(4), 249-263.

Corbett, G., and Leach, T. (1997). Southwest Pacific Rim gold-copper systems: Structure, alteration and mineralization. Short Course Manual. 318p.

Goldfarb, R.J., Baker, T., Dubé, B., Groves D.I., Hart C.J.R., and Gosselin, P. (2005). Distribution, character, and genesis of gold deposits in metamorphic terranes. Economic Geology, 100th Anniversary, 407-450.

Groves, D.I, Goldfarb, R.J., Robert, F., and Hart, J.R. (2003). Gold deposits in metamorphic belt: Overviews of current understanding, outstanding problems, future research and exploration significance. Economic Geology, 98(1), 1-29. doi: 10.2113/gsecongeo.98.1.1.

Groves, D.I., Goldfarb, R.J., Gebre-Mariam, M., Hagemann, S.G., and Robert, F. (1998). Orogenic gold deposits: a proposed classification in the context of their crustal distribution and relationship to other gold deposit types. Ore Geology Reviews, 13(1-5), 7-27. doi: 10.1016/ S0169-1368(97)00012-7.

Haeberlin, Y. (2002). Geological and Structural Setting, Age, and Geochemistry of the Orogenic Gold Deposits at the Pataz Province, Eastern Andean Cordillera, Peru. Ph.D. Thesis. Université de Genève, Switzerland.

Haeberlin, Y., Moritz, R., Fontbote, L., and Cosca, M. (2004). Carboniferous orogenic gold deposits at Pataz, eastern Andean Cordillera, Peru: geological and structural framework, paragenesis, alteration, and ${ }^{40} \mathrm{Ar} /{ }^{39} \mathrm{Ar}$ geochronology. Economic Geology, 99(1), 73-112.

Hart, C.J.R. (2005). Classifying, distinguishing and exploring for intrusion related gold systems. The Gangue: Newsletter of the Geological Association of Canada Mineral Deposits Division, 87, 1-9.

Irvine, T.N., and Baragar, W.R.A. (1971). A guide to the chemical classification of the common volcanic rocks. Canadian Journal of Earth Sciences, 8(5), 523-548. doi: 10.1139/e71-055.

Jia, Y., Kerrich, R., and Goldfarb, R. (2003). Metamorphic origin of ore-forming fluids for orogenic gold-bering quartz vein system in the North American Cordillera: Constraints from a reconnaissance study of $\delta^{15} \mathrm{~N}, \delta \mathrm{D}$ and $\delta^{18} \mathrm{O}$. Economic Geology, 98(1), 109-123. doi: 10.2113/ gsecongeo.98.1.109.

Klein, E.L., Harris, C., Giret, A., Moura C., and Angelica R.S. (2005). Geology and stable isotope $(\mathrm{O}, \mathrm{H}, \mathrm{C}, \mathrm{S})$ constraints on the genesis of the Cachoeira gold deposit, Gurupi Belt, northern Brazil. Chemical Geology, 221(3-4), 188-206. doi: 10.1016/j.chemgeo.2005.05.003.

Kreuzer, O.P. (2006). Textures, paragenesis and wall-rock alteration of lode-gold deposits in the Charters Towers district, north Queensland: 
implications for conditions of ore formation. Mineralium Deposita, 40, 639-663. doi: 10.1007/ s00126-005-0010-1.

Lambert, S.J., and Epstein, S. (1980). Stable isotope investigations of an active geothermal system in Valles Caldera, Jemez Mountains, New Mexico. Journal of Volcanology and Geothermal Research, 8(1), 111-129. doi: 10.1016/03770273(80)90010-4.

Leal-Mejía, H. (2011). Phanerozoic gold metallogeny in the Colombian Andes: A tectono-magmatic approach. Ph.D. Thesis. Universidad de Barcelona, España.

Londoño, C., Montoya, J.C., Ordoñez, O., y Restrepo, J.J. (2009). Características de las mineralizaciones vetiformes en el Distrito Minero Bagre-Nechí, Antioquia. Boletín de Ciencias de la Tierra, 26, 29-38.

Maniar, P.D., and Piccoli, P.M. (1989). Tectonic discrimination of granitoids. Geological Society of America Bulletin, 101(5), 635-643. doi: 10.1130/0016-7606(1989)101<0635:TDOG>2.3. CO;2.

McCuaig, T.C., and Kerrich, R. (1998). P-T-tdeformation-fluid characteristics of lode gold deposits: evidence from alteration systematics. Ore Geology Reviews, 12(6), 381-453. doi: 10.1016/S0169-1368(98)80002-4.

Middlemost, E.A. (1994). Naming materials in the magma/igneous rock system. Earth- Science Reviews, 37(3-4), 215-224. doi: 10.1016/00128252(94)90029-9.

Mikucki, E. (1998). Hydrothermal transport and depositional processes in Archaean lode-gold systems: a review. Ore Geology Reviews, 13(1-5), 307-321. doi: 10.1016/S0169-1368(97)00025-5.

Moritz, R. (2000). What have we learn about orogenic lode gold deposits over the past 20 years?. Scientific Communication. Section des Sciences de la Terre. University of Geneva, Switzerland. p. 1-7.

Naranjo-Sierra, E., Alvaran-Echeverri, M., y ZapataCardona, E. (2016). Análisis metalogenético preliminar del depósito vetiforme en mina La Ye, Antioquia-Colombia: características geológicas, isotópicas y estructurales. Revista Mexicana de
Ciencias Geológicas, 33(3), 316-328.

Ohmoto, H., and Goldhaber, M.B. (1997). Sulfur and carbon isotopes. In H.L. Barnes (Ed.), Geochemistry of hydrothermal ore deposits (pp. 517- 612), 3rd ed. New York: John Wiley \& Sons.

Owona, S., Ondoa, J.M., and Ekodeck, G.E. (2013). Evidence of quartz, feldspar and amphibole crystal plastic deformations in the paleoproterozoic Nyong Complex Shear Zones under Amphibolite to Granulite conditions (west Central African Fold Belt, SW Cameroon). Journal of Geography and Geology, 5(3), 186-201. doi: 10.5539/jgg. v5n3p186.

Pearce, J.A., Harris, N.W., and Tindle, A.G. (1984). Trace element discrimination diagrams for the tectonic interpretation of granitic rocks. Journal of Petrology, 25, 956-983.

Pirajno, F. (1992). Hydrothermal Mineral Deposits: Principles and fundamental concepts for the exploration geologist. Berlin: Springer-Verlag.

Qiu, Y., Groves, D.I., McNaughton, N.J., Wang, L., and Zhou, T. (2002). Nature, age and tectonic setting of granitoid-hosted, orogenic gold deposits in the Jiaodong Peninsula, eastern North China craton, China. Mineralium Deposita, 37(3-4), 283-305. doi: 10.1007/s00126-001-0238-3.

Restrepo, J.J., and Toussaint, J.F. (1988). Terranes and continental accretion in the Colombian Andes. Episodes, 11(3), 189-193.

Ridley, R.J., and Diamond, L. (2000). Fluid chemistry of orogenic lode gold deposits and implications for genetic models. SEG Reviews, 13, 141-162.

Rimstidt, J.D. (1997). Gangue mineral transport and deposition. In H.B. Barnes (Ed.), Geochemistry of hydrothermal ore deposits, (pp. 487-515). John Wiley \& Sons.

Rollinson, H. (1993). Using geochemical data: evaluation, presentation, interpretation. London: Longman.

Rye, R.O. (2005). A review of the stable-isotope geochemistry of sulfate minerals in selected igneous environments and related hydrothermal systems. Chemical Geology, 215(1-4), 5-36. doi: 10.1016/j.chemgeo.2004.06.034. 
Seal II, R.R. (2006). Sulfur isotope geochemistry of sulfide minerals. Reviews in Mineralogy and Geochemistry, 61(1), 633-677. doi: 10.2138/ rmg.2006.61.12.

Shaw, R.P. (2000). Gold mineralization in the Northern Andes: magmatic setting vs. metallogeny. XI International Mining Congress, Bogotá.

Sheppard, S.M. (1981). Stable isotope geochemistry of fluids. Physics and Chemistry of the Earth, 13-14, 419-445.

Sillitoe, R.H. (2008). Major gold deposits and belts of the North and South American Cordillera: Distribution, tectonomagmatic settings, and metallogenic considerations. Economic Geology, 103(4), 663-687. doi: 10.2113/ gsecongeo.103.4.663.

Sillitoe, R.H., and Thompson, J.F.H. (1998). Intrusionrelated vein gold deposits: types, tectonomagmatic settings and difficulties of distinction from orogenic gold deposits. Resource Geology, 48(4), 237-250. doi: 10.1111/j.1751-3928.1998. tb00021.x.

Spikings, R., Cochrane, R., Villagomez, D., Van der Lelij, R., Vallejo, C., Winkler, W., and Beate, B. (2015). The geological history of northwestern South America: from Pangaea to the early collision of the Caribbean Large Igneous Province (290-75 Ma). Gondwana Research, 27(1), 95-139. doi: 10.1016/j.gr.2014.06.004.

Starling, A. (2014). Structural review of La Ye mine and District. Internal Field Report prepared for Mineros S.A and Operadora Minera S.A.S.

Starling, A. (2015). Structural review of La Ye and Icacales-Los Mangos mines. Internal Field Report prepared for Mineros S.A and Operadora Minera S.A.S.

Stipp, M., Stünitz, H., Heilbronner, R., and Schmid, S.M. (2002). The eastern Tonale fault zone: a 'natural laboratory' for crystal plastic deformation of quartz over a temperature range from 250 to 700 ${ }^{\circ}$ C. Journal of Structural Geology, 24(12), 18611884. doi: 10.1016/S0191-8141(02)00035-4.

Takagi, T., and Tsukimura, K. (1997). Genesis of oxidized and reduced-type granites. Economic Geology, 92(1), 81-86. doi: 10.2113/ gsecongeo.92.1.81.
Taylor, H.P. (1974). The application of oxygen and hydrogen isotope studies to problems of hydrothermal alteration and ore deposition. Economic Geology, 69(6), 843-883. doi: 10.2113/ gsecongeo.69.6.843.

Taylor, H.P. (1997). Oxygen and hydrogen isotope relationships in hydrothermal mineral deposits. In H.L. Barnes (Ed.), Geochemistry of hydrothermal ore deposits (pp. 229-302), 3rd ed. New York: John Wiley \& Sons.

Ueda, A., and Krouse, H.R. (1986). Direct conversion of sulphide and sulphate minerals to $\mathrm{SO}_{2}$ for isotope analyses. Geochemical Journal, 20, 209-212.

Varona-Bravo, D.S., Naranjo-Sierra, E., y Toro, L.M. (2016). Características geoquímicas y petrográficas del stock El Carmen en el distrito minero El Bagre. Reporte Interno. Operadora Minera S.A.S.

Vinasco, C.J., Cordani, U.G., González, H., Weber, M., and Pelaez, C. (2006). Geochronological, isotopic, and geochemical data from PermoTriassic granitic gneisses and granitoids of the Colombian Central Andes. Journal of South American Earth Sciences, 21(4), 355-371. doi: 10.1016/j.jsames.2006.07.007.

Edwin Naranjo-Sierra

ORCID: 0000-0002-8634-5370

Mauricio Alvarán-Echeverri

ORCID: 0000-0001-5097-9761

Trabajo recibido: abril 12 de 2017

Trabajo aceptado: noviembre 20 de 2017 